\title{
Structural variations of the cathode deposit in the carbon arc
}

\author{
Yao-Wen Yeh ${ }^{\mathrm{a}, \mathrm{b}^{*}}$, Yevgeny Raitses ${ }^{\mathrm{a}}$, and Nan Yao ${ }^{\mathrm{b}}$
}

${ }^{a}$ Princeton Plasma Physics Laboratory, Princeton University, P.O. Box 451, Princeton, NJ 08543, USA

${ }^{\mathrm{b}}$ Princeton Institute for Science and Technology of Materials, Princeton University, Princeton, NJ 08544, USA

\begin{abstract}
Synthesis of various carbon nanostructures, including fullerenes, single-walled and multiwalled nanotubes and nanoparticles, by arc discharges relies on ablation of the graphite anode and deposition of synthesized carbonaceous products on the cathode surface and on the reactor chamber walls. For backbone all-carbon system, the cathode deposit plays a critical role in sustaining the arc discharge and thereby, the synthesis processes. This deposit usually exhibits spatially distinct structural variations with three different axially symmetrical morphologies. In particular, a rim of pyrolytic carbon separates the innermost core consisting of multi- walled carbon nanotubes from the outmost ring with powdery amorphous carbon soot. Experiments revealed a strong correlation between the current conducting arc attachment to the cathode deposit and the nanotube forming area in the deposit. Results suggest that particle and heat fluxes from the plasma are responsible for purity of nanotubes in this deposit core area. It appears that a better synthesis selectivity can be obtained in low ablation regime which is characterized by a nearly constant arc current density independent on the anode diameter.
\end{abstract}

\section{Introduction}

DC and AC arc discharges at moderate (1-100 Torr) and atmospheric pressure levels are commonly used for synthesis of carbon nanostructures, including fullerenes, nanotubes and nanoparticles[1, 2, 3, 4], and other nanomaterials such as $\mathrm{ZnO}$ [5], $\mathrm{SiC}$ [6], and gallium oxide[7], etc. In a typical synthesis by a DC arc discharge (so-called anodic arc), the anode electrode is consumed by ablation to provide a feedstock of atoms and molecules to sustain nucleation and growth of nanostructures. Ablated and synthesized products are then deposited on the electrodes and the reactor chamber wall. Independent of the material of interest, the cathode deposit is a common feature for all anodic arc discharges. This plasma facing deposit plays an important role in sustaining the arc discharge as it acts as an effective cathode electrode. For carbon arc discharges, the deposit has usually a complex morphology with various synthesized structures including fullerenes, multi-walled nanotubes, and amorphous carbon soot[2, 8, 9, 10, 11]. In this

* Corresponding author.

E-mail address: yyeh@princeton.edu (Yao-Wen Yeh) 
paper, we demonstrate that these structural variations of the cathode deposit are associated with spatial variations of plasma, particle, and heat fluxes at the arc attachment to the deposit. These results can be generalized for low and high anode ablation modes, which are usually observed for synthesis arcs[12, 13, 14, 15]. Recent theoretical models attributed these ablation modes to the formation of the negative and positive anode sheath regimes of the arc, respectively. It was also shown that power flux from the plasma to the cathode depends strongly on the anode sheath regimes[12, 16]. We show now that structural variations of the cathode deposit depend also on the anode ablation mode.

The measured correlation between arc properties and carbon structures on the cathode deposit is a key result of this paper, which goes beyond previous studies of structural variations of the arc deposit on the cathode[13]. The paper is organized as follows: in Section 2, we describe the experimental setup for the characterization of the current and the surface temperature distributions at the cathode. Experimental results are described and analyzed in Section 3. Conclusions of this study of synergetic plasma and material processes and practical implications of the results are summarized in Section 4.

\section{Experimental}

The experimental arc synthesis setup (Fig. 1) employed in the present study was described elsewhere[13]. The anode (A) and cathode (C) are set up in a vertical configuration in order to avoid asymmetrical effects on the arc and deposition brought by convective flow. In order to measure the arc current distribution, a segmented cathode composed of a graphite central electrode (diameter $d=3.2 \mathrm{~mm})$ and a copper rim electrode $(\mathrm{d}=50.8 \mathrm{~mm})$ is used. In order to allow a better measurement resolution of the current distribution and its correlation with the deposit morphology, the diameter of the central electrode is almost twice smaller than in Ref.[13]. The rim electrode is insulated from the central electrode by a thin layer of boron nitride with a thickness of $0.5 \mathrm{~mm}$ in order to minimize the disturbance to the arc. The central and rim electrodes are connected electrically to the arc power supply through separate shunt resistors of 2 $\mathrm{mOhm}$ and $1 \mathrm{mOhm}$, respectively. In arc operation, these resistors are used to measure the currents the segmented electrodes. 

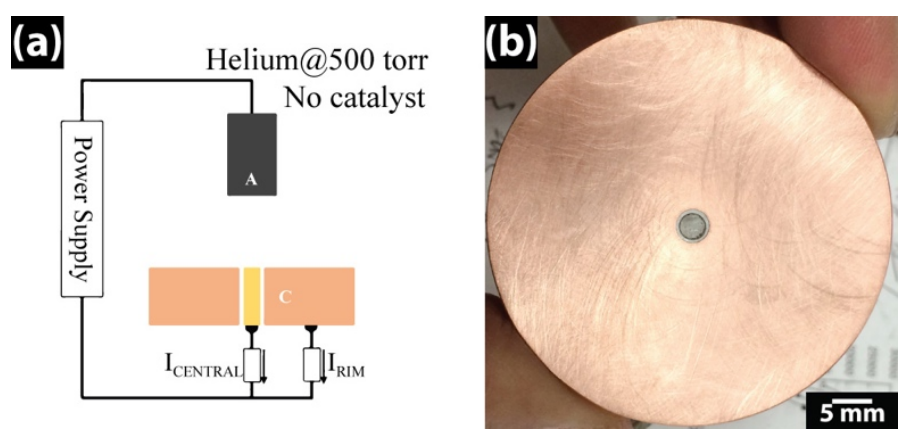

Fig. 1: (a) A schematic of the arc setup with a graphite anode (A) and the segmented cathode (C). (b) Photograph of the segmented cathode.

The carbon arc discharge experiments were conducted in a reactor chamber filled with helium gas at 500 Torr. The arc was initiated by bringing the anode into contact with the cathode, after which the current was maintained at $65 \mathrm{~A}$. An external control system increased the electrode gap until the specified discharge voltage was reached. The gap was about $2 \mathrm{~mm}$ wide. Throughout all experiments described in this paper, the voltage measured across the two electrodes was maintained about $20 \mathrm{~V}$. This voltage includes the voltage drop across the arc and along the electrodes[12].

After the arc initiation, it usually took about $10 \mathrm{~s}$ for the arc current to stabilize and for the anode to reach the specified inter-electrode gap. During the arc operation, the graphite anode is ablated, producing flux of carbon atoms, molecules and clusters. The arc plasma is mainly generated from these carbon species some of which are ionized producing positive atomic and molecular ions[17]. The fluxes of charged and neutral plasma particles to the cathode form a carbonaceous deposit (Fig. 2). In present experiments, the arc was operated with segmented and non-segmented cathodes made from graphite and copper. We found no difference in structures and properties of the cathode deposits formed with these different cathodes.
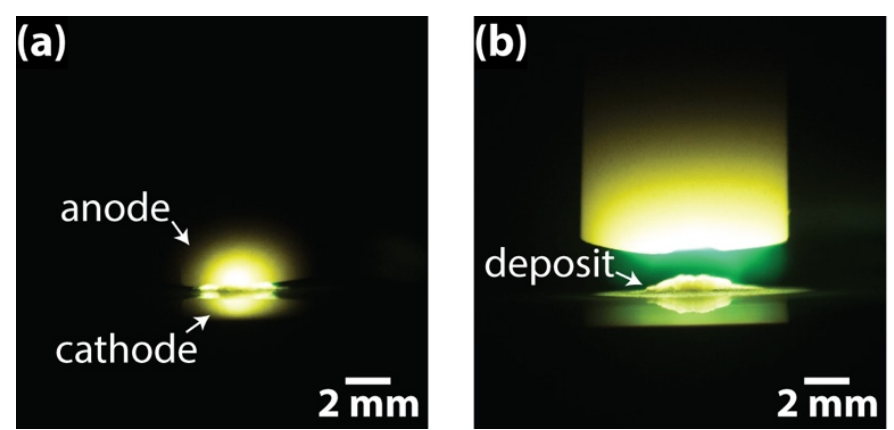

Fig. 2: Unfiltered images of (a) the onset of the arc and (b) the arc steady state. The top and bottom electrodes are the anode and the cathode, respectively. The small hump of the material on the cathode is the carbonaceous deposit. 
Reliable measurements of the current distribution using the segmented cathode were feasible only for a steady-state arc operation in the low ablation mode. In this mode, the deposit grows relatively slowly (about $30 \mathrm{~s}$ ) before it completely electrically shorts the segmented electrodes. In the high ablation mode, the deposit was large enough to short the electrodes while arc was still reaching a steady state operation (i.e. less than $10 \mathrm{~s}$ ). From previous arc studies[12, 14], an arc current of $65 \mathrm{~A}$ and an anode diameter of $9 \mathrm{~mm}$ were selected to facilitate the low ablation mode. From the measurements of the anode weight and the cathode deposit thickness before and after arc operation, an ablation of $0.56 \pm 0.03 \mathrm{mg} / \mathrm{s}$ and a deposit thickness of about 1 $\mathrm{mm}$ were determined. This is consistent and comparable with ablation rates measured for this regime in Refs.[12, 13].

In addition, a set of arc experiments was also conducted in the high ablation mode, using a $6 \mathrm{~mm}$ diameter graphite anode and the same operation conditions (arc current, voltage, and helium gas pressure) as for the low ablation mode. The main purpose of these experiments was to study the effect of the anode ablation mode on the morphology of the cathode deposit.

The distribution of the surface temperature of the cathode deposit was also measured during the arc discharge. For these measurements, a calibrated FLIR tau 2640 infrared camera together with a $3.2 \%$ transmittance neutral density filter were placed to acquire the side view of the electrodes and cathode deposit. Similar to the measurement procedure reported earlier[13, 14], C-type thermocouples inserted into the anode and cathode were used to calibrated this IR camera. The temperature measured by the IR camera had an uncertainty of about $100{ }^{\circ} \mathrm{C}$. Moreover, the same thermocouples were used in order to determine the heat flux to the electrodes.

The cathode deposits produced in arc experiments were carefully studied via electron microscopy. The cathode deposit from each run was kept on the cathode without disturbance and first surveyed by an FEI Quanta 200 field emission SEM operated at $10 \mathrm{kV}$. This survey allowed us to identify characteristic parts of the deposit with distinguished appearances. After that, these parts were separately collected, sonicated in an acetone bath for about 3 minutes, and dispersed onto a TEM grid. The TEM samples were studied by a Philip CM200 field emission TEM at 200 $\mathrm{kV}$. Structural analysis of the deposit was carried out using a Rigaku MiniFlex XRD with Cu Ka 
as the X-ray source.

\section{Results and discussion}

\subsection{Cathode deposit}

\subsubsection{Microscopic evaluation}

A cathode deposit occupies usually a large area with an effective diameter larger but comparable with the anode diameter. For example, for a $9 \mathrm{~mm}$ diameter anode, including the outer soot formation the deposit is about $14 \mathrm{~mm}$. Fig. 3 demonstrates three typical regions of the cathode deposits collected in the experiments. Each region features distinctive structures compared to one another. Starting from the innermost region to the outermost region, the deposit consists of (1) a fibrous core that contains loosely entangled and randomly oriented multi-walled carbon nanotubes and polyhedral nanoparticles, (2) a particulate rim that contains curvy pyrolytic carbon sheets, and (3) amorphous carbon soot. Nanotubes were found only in the core area of the deposit which is typically much smaller than the total deposit area. For example, for a $9 \mathrm{~mm}$ diameter anode and $14 \mathrm{~mm}$ diameter cathode deposit, the diameter of the core area is about $3 \mathrm{~mm}$.

While the morphologies of the thin pyrolytic carbon rim appear similarly to the outermost amorphous soot, subsequent TEM and XRD characterizations revealed structural differences. Detailed electron images of the fibrous core, pyrolytic carbon rim, and amorphous carbon are shown in Figures 3b, 3c, and 3d, respectively. The fibrous core and the particulate rim accounted for about $60 \%$ of the ablated anode mass.

Although three distinct regions of the cathode deposit were previously reported [8], the present study focuses on a correlation between structural variations in these deposit regions with spatial variations of the arc discharge at its attachment to the cathode. In addition, a separate set of arc experiments with a small $3 \mathrm{~mm}$ diameter cathode, it was confirmed that these three regions of the cathode deposit are only formed when the cathode diameter is larger than the anode diameter. In fact, the deposit found on the small diameter cathode had only multi- walled carbon nanotubes and nanoparticles without the presence of pyrolytic carbon rim and amorphous soot. 

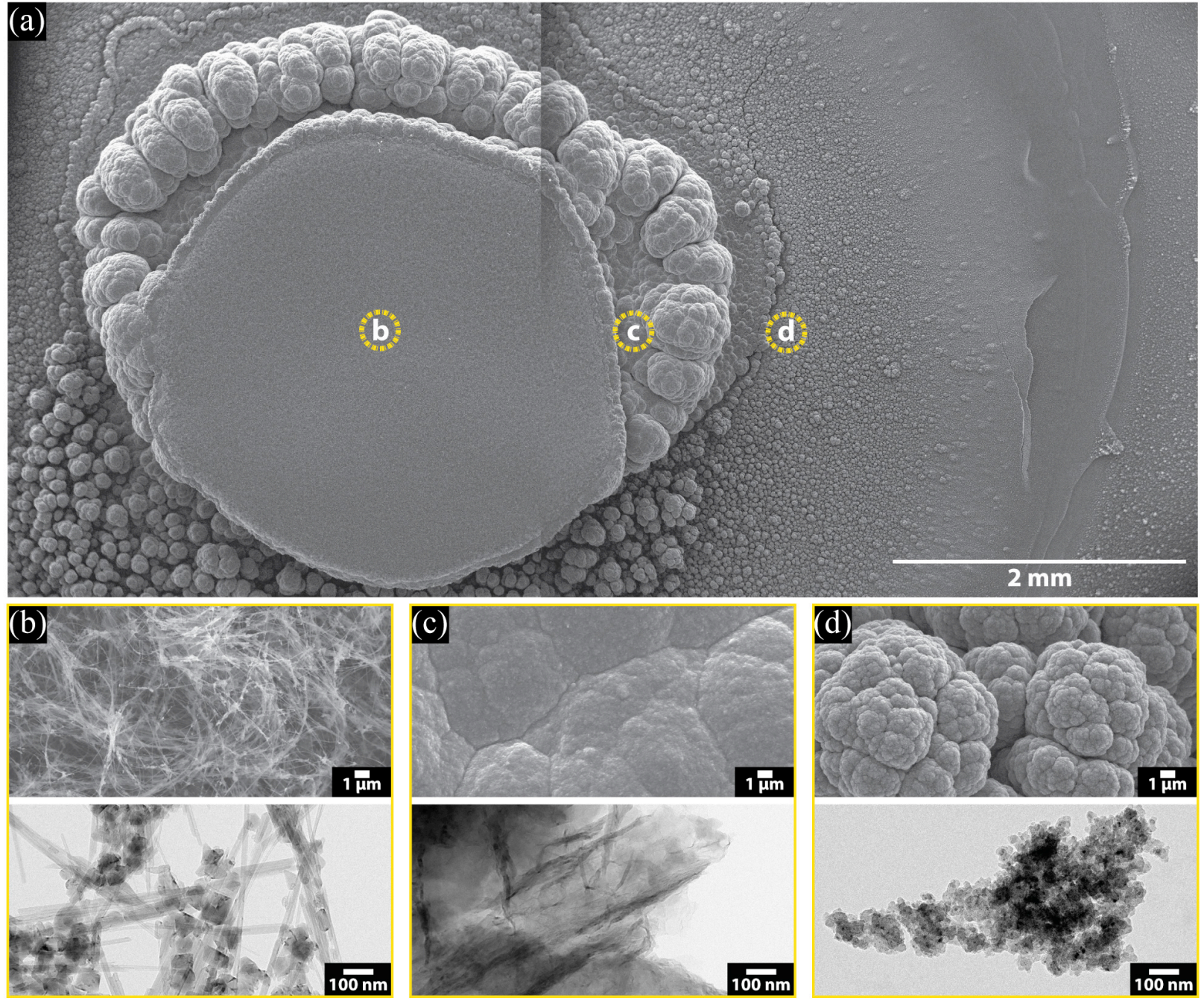

Fig. 3: Electron micrographs of the cathode deposit: (a) three regions of the deposit with three different morphologies, (b) the fibrous core that contains multi-walled carbon nanotubes and nanoparticles only, (c) particulate rim that contains pyrolytic carbon sheets, and (c) amorphous carbon soot.

\subsubsection{Structural analysis by XRD}

The structural characterization of the cathode deposit and the starting graphite materials were carried out by Rigaku MiniFlex XRD. As shown in Fig. 4, while the deposit core and rim materials consist of different morphologies, they show the same turbostratic graphitic structure, with the absence of c-axis ordering indicated by the missing (101) and (112) peaks that are observed from the starting anode material[11, 18]. Both the core and rim materials show (002) interlayer spacing to about $3.4 \AA$. The full-width-half-maxima (FWHM) of the (002) peaks are 0.6 and 0.9 degrees for the deposit core and rim materials, respectively. The narrower (002) FWHM observed in the core material indicates that the core has higher crystallinity than the rim 
material, which in turn suggests that the forming temperature of the core was higher than the rim. As will be discussed later the observation agrees with the deposit temperature measurement during the discharge.

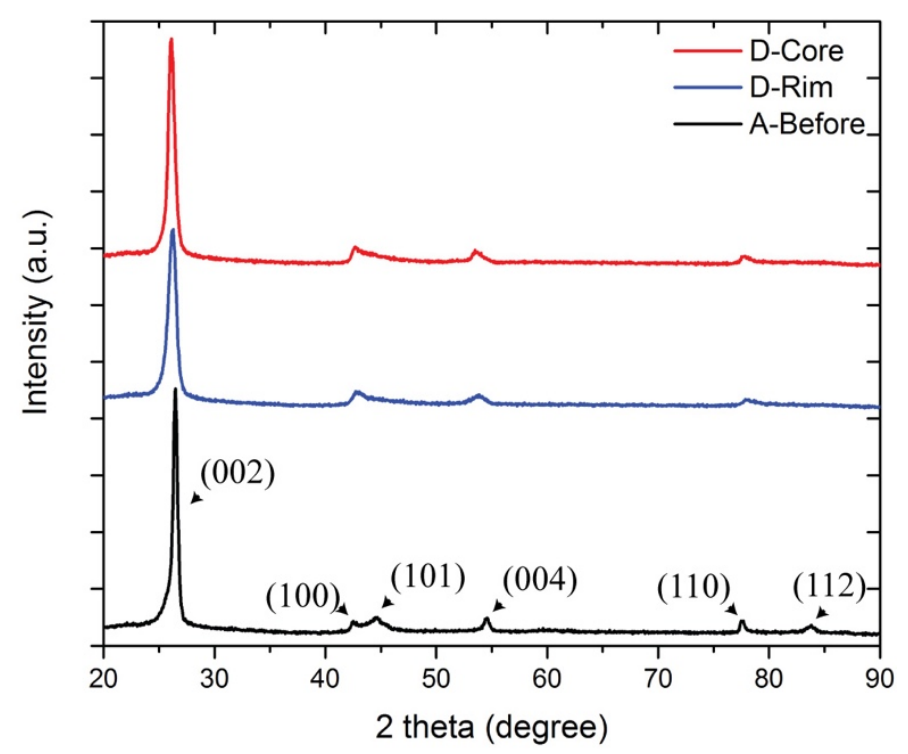

Fig. 4: XRD patterns for the fibrous core of the deposit (D-core) in red, the pyrolytic carbon rim (D-Rim) in blue, and the starting graphite anode (A-Before) in black. Note that the c-axis ordering peaks (101) and (112) peaks are only observed from the starting anode material not from the deposit material.

\subsubsection{Arc current distribution}

Fig. 5 shows the measured currents through both segmented electrodes during the arc operation. Experiments revealed that about $95 \%$ of the total arc current is conducted through the central segmented-electrode (Fig. 5a). The cathode deposit formed on this electrode contains multi-walled carbon nanotubes and particles (Fig. 5b). The remaining larger part of the cathode deposit does not have these nanostructures.

Note that in some arc runs, the arc attachment would shift away from its initial position during the electrode separation. This is likely due to a combination of the arc transient processes and mechanical motion of the arc electrodes. As the result of the shift, the currents of the central and rim electrodes were comparable (Fig. 5c). Moreover, the center of the deposit would also shift with the arc attachment (Fig. 5d). Nevertheless, the diameter of the deposit core region containing multi-walled carbon nanotubes remained roughly the same, about $3 \mathrm{~mm}$. This result 
appears to be consistent with the above correlation between the area of the arc attachment to the cathode and the deposit area with the nanotubes.
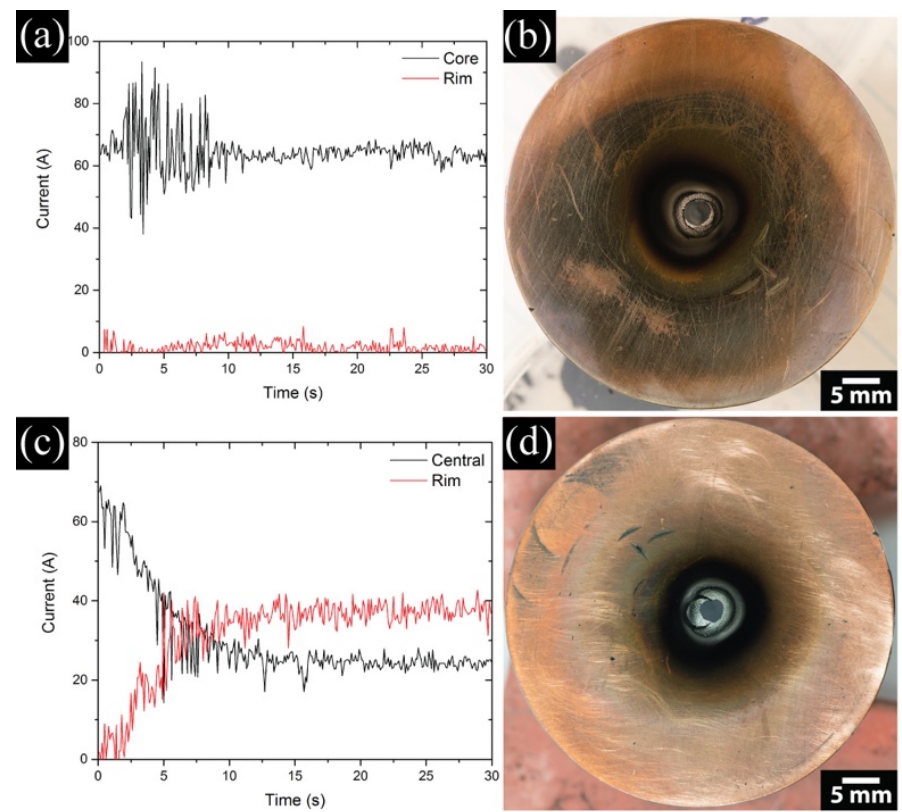

Fig. 5: Carbon arc with segmented cathode: (a) currents through the core and rim of electrodes. (b) The segmented cathode with the cathode deposit after discharge. As the result of arc attachment shift, (c) the currents of the central and rim electrodes were comparable and (d) the center of the deposit would also shift with the arc attachment.

\subsubsection{Surface temperature}

Results of IR measurements of surface temperature of the cathode deposit during the arc discharge are shown in Fig. 6 and 7. The surface temperature reaches its maximum of about 3500 $\mathrm{K}$ at the center of the cathode deposit above the core electrode (Fig. 6). The average temperature of the deposit across this electrode region is about $3100 \mathrm{~K}$.

Correlating with the deposit structural features, IR measurements suggest that the nanotubes form and withstand the temperatures between $2700 \mathrm{~K}$ and $3500 \mathrm{~K}$ (Fig. 6 and 7). The lower bound of the observed temperature range is similar to the value reported by Liang et al.[19] who studied the nanotube-forming temperature at the anode surface during the arc. A relatively strong temperature decrease at a distance of $1.5 \mathrm{~mm}$ from the center (Fig. 7) corresponds to the boundary between the multi-walled carbon nanotube core and the pyrolytic rim (Fig. 6b). This temperature drop should be due to a non-uniform radial profile of the heat flux from the plasma predicted by the arc model of Ref.[16]. 

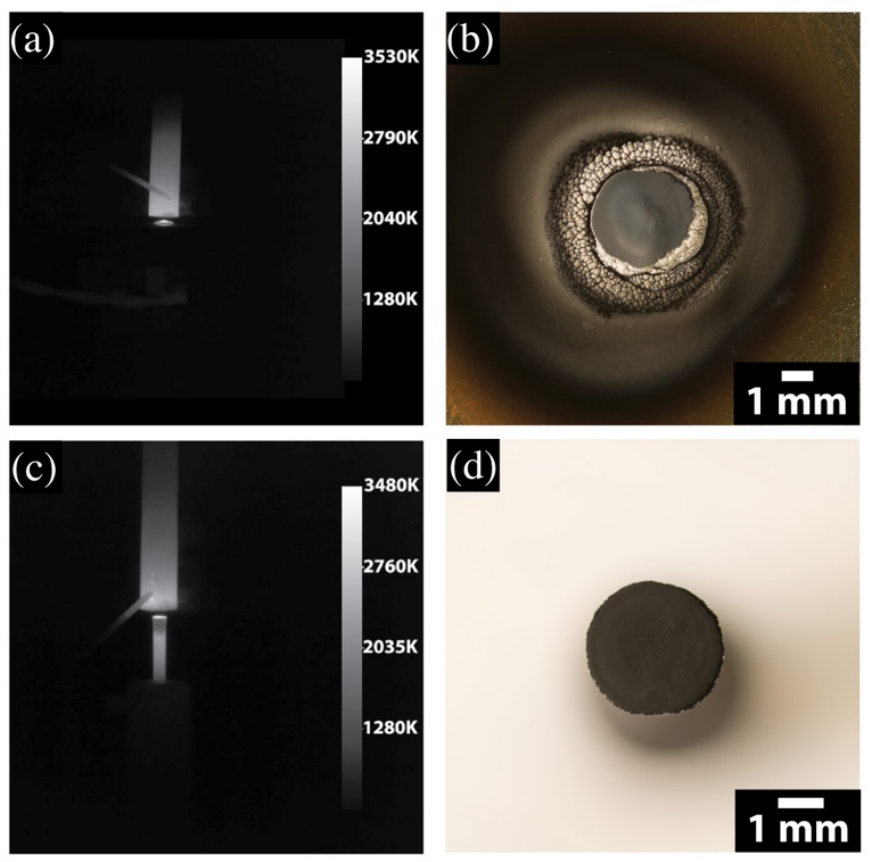

Fig. 6. Thermographs and deposit materials for $(a, b)$ the segmented cathode and $(c, d)$ the $d=$ $3.2 \mathrm{~mm}$ graphite cathode.

3.2 Analysis of correlations between plasma fluxes and deposit structure

The cathode deposit accumulates more material at the center to form a hump on the cathode (Fig. 2b). Huczko et al.[20] reported that for fullerene synthesis condition (helium at 100 Torr), both the temperature and the density of carbon radicals (e.g. $C_{2}$ ) in the arc column have their maximum values at the center of the plasma column. While the helium pressure used in the present study is 500 Torr, the observed profile of the cathode deposit can be attributed to nonuniform distributions of particle and heat fluxes from the plasma leading to the humped profile of the deposit with a higher surface temperature at the hump region (Fig. 7). 


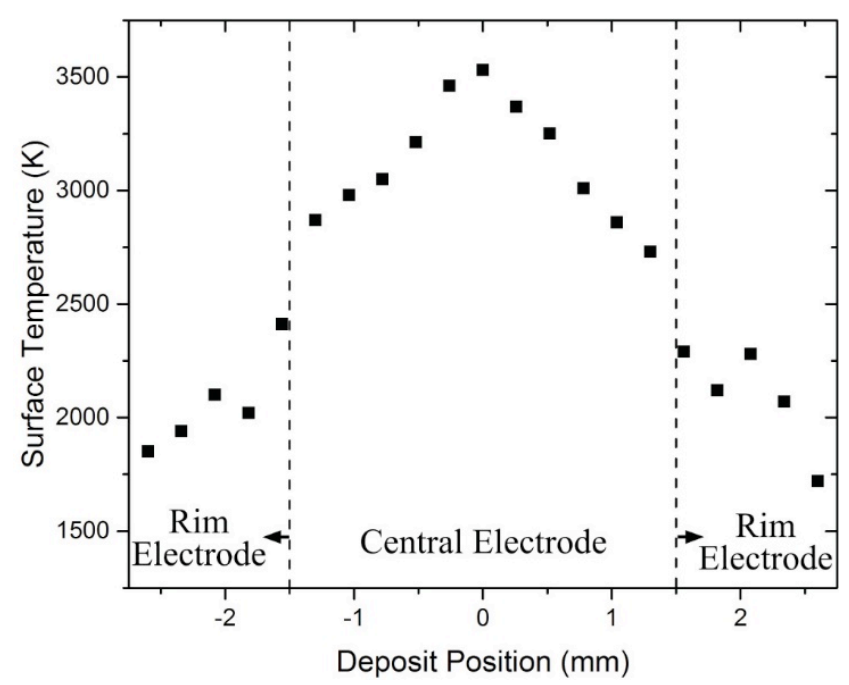

Fig. 7: The radial profile of the surface temperature of the carbonaceous cathode deposit during the arc discharge. Experiments were conducted with segmented cathode electrode. The dashed vertical lines indicate the boundary between nanotube forming core (the central electrode) and the pyrolytic carbon and soot depositing area (the rim electrode).

Similar to the low ablation mode, the high ablation mode produces three regions of the cathode deposit with similar structural variations, including the fibrous core region with multiwalled carbon nanotubes. The surface temperature also peaks in this region and reaches about $3500 \mathrm{~K}[13]$. However, for the high ablation mode, the diameter of the core region is larger than the diameter of the core region measured for the low ablation mode. Assuming that for this ablation mode, the arc current is also conducted mainly through this fibrous core of the deposit, the increases of the core diameter translates to the decrease of the current density through the cathode deposit. The current densities through the core region are 9.2 and $3.2 \mathrm{~A} / \mathrm{mm}^{2}$ for low and high ablation modes, respectively.

Although the decrease of the current density implies the reduction of the power flux density from the plasma to the cathode, the maximum deposit surface temperature is comparable with the deposit temperature measured for the low ablation mode. Following modeling results of Ref. [16], this result can be explained by the abundant latent heat, which is carried by carbon feedstock materials from the plasma to the cathode. According to predictions of Refs. [14] and [16], the contribution of the latent heat to the energy balance at the cathode is larger for the high ablation mode than for the low ablation mode. Therefore, in spite of a lower current density for high ablation mode, both ablation modes deliver sufficient heat from the plasma to maintain similar surface temperatures. 
Importantly, for the high ablation mode, the deposit core contains significantly more carbon nanoparticles than for the low ablation mode (Fig. 8). Based on the SEM images, the tube-to-particle ratios are estimated to be 0.8 and 6.8 for the high and low ablation modes, respectively. This observation may suggest that for the high ablation mode, the carbon feedstock to the cathode is too large to be fully transformed into nanotube structures at the measured deposit temperatures. Thus, the selectivity of carbon nanotubes synthesis measured as the ratio of tube to particle is larger for the low ablation mode. 

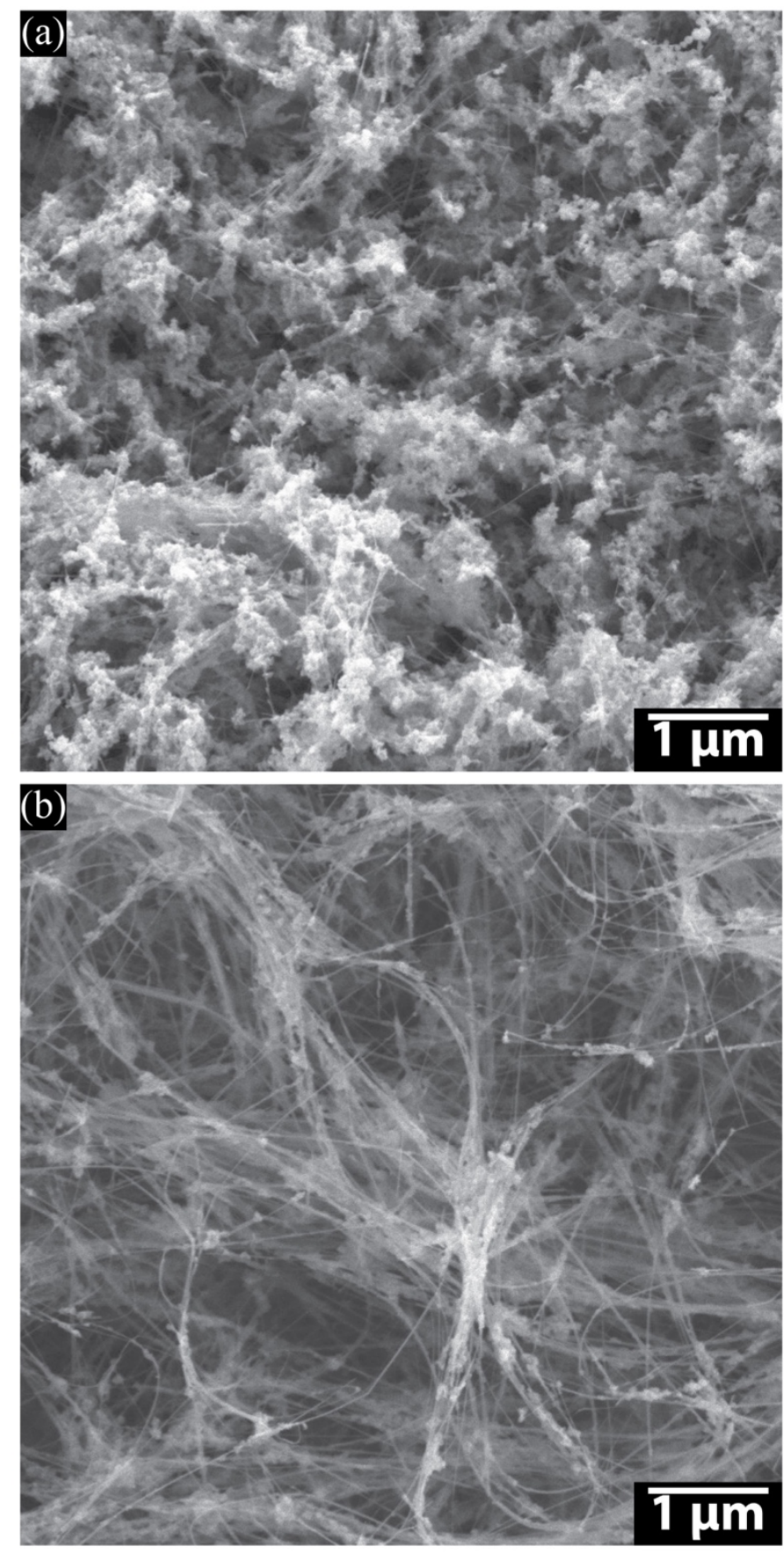

Fig. 8: SEM images of the fibrous core obtained from (a) high and (b) low ablation modes.

Note that similar results were obtained in a separate set of low ablation experiments in which the anode diameters of $9 \mathrm{~mm}, 12 \mathrm{~mm}$, and $14 \mathrm{~mm}$ were used while keeping the current constant at 65 A. For the low ablation operation, the current density over the deposit core area was nearly independent of the anode diameter. In this regime, the synthesis selectivity was also better than that for high ablation mode obtained with $6 \mathrm{~mm}$ anode. 


\section{Conclusion}

Arc synthesis of various carbon nanostructures, including fullerenes, single-walled and multi-walled nanotubes and nanoparticles involve the ablation of the graphite anode and the deposition of synthesized products on the cathode surface and on the reactor chamber walls. It is shown that the carbon cathode deposit consists of three different regions with different morphologies, including (1) a core region at the center of the deposit with multi-walled carbon nanotubes enclosed by (2) a particulate pyrolytic carbon rim, which is surrounded by (3) amorphous carbon soot. Measurements of the arc current at the segmented cathode and the surface temperature of the cathode deposit reveal strong correlation between the radial distribution of the arc current, the surface temperature, and variations of structural morphology of the deposit. In particular, it is shown that almost the entire current is conducted through the core region with multi-walled carbon nanotubes. This correlation is consistent with the maximum surface temperature and the humped profiled of the cathode deposit measured for the arc discharge operating in low and high ablation modes. According to the surface temperature measurements, multi-walled carbon nanotube formed in the deposit core survive temperatures between $2700 \mathrm{~K}$ and $3500 \mathrm{~K}$.

While a higher anode ablation rate results in a higher cathode deposition rate, more carbon nanoparticles are also formed for the arc discharge operating with high anode ablation. Thus, there should be an optimal balance between the temperature and the carbon particle flux to the cathode for the maximum nanotube synthesis yield. From these experiments, this balance is realized when the arc operates in the low ablation mode with a constant current density at the deposit core, i.e. independent of the anode diameter. It is in the low ablation mode that recent theoretical models predicted a recycling of the deposit material by the arc plasma[16]. In this process, the cathode deposit evaporates producing a flux of carbon atoms and molecules from the deposit surface to the plasma. These evaporated particles are ionized in the sheath between the cathode and the plasma. Then, the resulting ions are accelerated back to the cathode. It would be interesting to explore if this self-organized plasma-material process could be responsible for a smaller fraction of carbon nanoparticles obtained in this arc regime.

The above results and conclusions are not unique to the described arc configuration. In fact, we conducted separate set of experiments with different carbon arc configurations, 
including the use of all graphite electrodes and different electrode dimensions. In these experiments, better synthesis selectivity was also obtained for low ablation mode of the graphite electrode.

\section{Acknowledgements}

The authors wish to thank Mr. Jonathan Ng and Mr. James Mitrani, Dr. Sophia Gershman, and Prof. Bruce Koel for fruitful discussions. We also thank Mr. Alex Merzheskiy for his technical support of this work. This work was supported by U.S. Department of Energy, Office of Science, Basic Sciences, Materials Sciences and Engineering Division and the National Science Foundation-MRSEC program through the Princeton Center for Complex Materials (DMR0819860).

\section{References}

[1] H. W. Kroto, J. R. Heath, S. C. O’Brien, R. F. Curl, R. E. Smalley, C60: Buckminsterfullerene, Nature 318 (6042) (1985) 162-163.

[2] S. Iijima, Helical microtubules of graphitic carbon, Nature 354 (6348) (1991) 56-58.

[3] C. Journet, M. Picher, V. Jourdain, Carbon nanotube synthesis: from large-scale production to atomby-atom growth, Nanotechnology 23 (14) (2012) 142001.

[4] P. J. Harris, Solid state growth mechanisms for carbon nanotubes, Carbon 45 (2) (2007) 229 - 239, ISSN 0008-6223, doi: http://dx.doi.org/10.1016/j.carbon.2006.09.023.

[5] F. Fang, J. Futter, A. Markwitz, J. Kennedy, UV and humidity sensing properties of ZnO nanorods prepared by the arc discharge method, Nan- otechnology 20 (24) (2009) 245502.

[6] T. Seeger, P. Kohler-Redlich, M. Ruhle, Synthesis of Nanometer- Sized SiC Whiskers in the ArcDischarge, Advanced Materials 12 (4) (2000) 279-282, ISSN 1521-4095, doi:10.1002/(SICI)15214095(200002)12:4¡279::AID-ADMA279;3.0.CO;2-1.

[7] Y. C. Choi, W. S. Kim, Y. S. Park, S. M. Lee, D. J. Bae, Y. H. Lee, G.-S. Park, W. B. Choi, N. S. Lee, J. M. Kim, Catalytic Growth of -Ga2O3 Nanowires by Arc Discharge, Advanced Materials 12 (10) (2000) 746-750, ISSN 1521-4095, doi:10.1002/(SICI)1521- 4095(200005)12:10;746::AIDADMA746;3.0.CO;2-N.

[8] P. M. Ajayan, P. Redlich, M. Rhle, Balance of graphite deposition and multishell carbon nanotube growth in the carbon arc discharge, Journal of Materials Research 12 (1997) 244-252, ISSN 2044-5326, doi: 10.1557/JMR.1997.0032.

[9] E. G. Gamaly, T. W. Ebbesen, Mechanism of carbon nanotube formation in the arc discharge, Phys. 
Rev. B 52 (1995) 2083-2089, doi: 10.1103/PhysRevB.52.2083.

[10] D. Tang, L. Sun, J. Zhou, W. Zhou, S. Xie, Two possible emission mechanisms involved in the arc discharge method of carbon nanotube preparation, Carbon 43 (13) (2005) 2812 - 2816, ISSN 0008-6223, doi: http://dx.doi.org/10.1016/j.carbon.2005.05.034.

[11] Y. Saito, T. Yoshikawa, M. Inagaki, M. Tomita, T. Hayashi, Growth and structure of graphitic tubules and polyhedral particles in arc-discharge, Chemical Physics Letters 204 (3) (1993) 277 - 282, ISSN 0009-2614, doi:http://dx.doi.org/10.1016/0009-2614(93)90009-P.

[12] A.J.Fetterman,Y.Raitses,M.Keidar,Enhancedablationofsmallanodes in a carbon nanotube arc plasma, Carbon 46 (10) (2008) 1322 - 1326, ISSN 0008-6223, doi:http://dx.doi.org/10.1016/j.carbon.2008.05.018.

[13] J. Ng, Y. Raitses, Role of the cathode deposit in the carbon arc for the synthesis of nanomaterials, Carbon 77 (2014) 80 - 88, ISSN 0008-6223, doi:http://dx.doi.org/10.1016/j.carbon.2014.05.007.

[14] J. Ng, Y. Raitses, Self-organisation processes in the carbon arc for nanosynthesis, Journal of Applied Physics 117 (6) 063303, doi: http://dx.doi.org/10.1063/1.4906784.

[15] D. Delaportas, P. Svarnas, I. Alexandrou, A. Siokou, K. Black, J. W. Bradley, Al2O3 nanoparticle production by arc-discharge in water: in situ discharge characterization and nanoparticle investigation, Journal of Physics D: Applied Physics 42 (24) (2009) 245204.

[16] V. A. Nemchinsky, Y. Raitses, Atmospheric pressure arc discharge with ablating graphite anode, Journal of Physics D: Applied Physics 48 (24) (2015) 245202.

[17] V. Vekselman, M. Feurer, Y.-W. Yeh, B. Stratton, Y. Raitses, Optical Emission Spectroscopy of Arc Discharge for Nanomaterial Synthesis, APS Division of Plasma Physics, Savannah, Georgia, 2015.

[18] M. S. D. G. Dresselhaus, Carbon nanotubes synthesis, structure, properties, and applications, Springer-Verlag, 2001.

[19] F.Liang, M. Tanaka, S. Choi, T. Watanabe, Investigation of Carbon Nanomaterials Growth on Anode Surface by Arc Discharge Method, JOURNAL OF CHEMICAL ENGINEERING OF JAPAN 47 (3) (2014) 296-300, doi:10.1252/jcej.13we174.

[20] A. Huczko, H. Lange, P. Byszewski, M. Poplawska, A. Starski, Fullerene Formation in Carbon Arc: Electrode Gap Dependence and Plasma Spectroscopy, The Journal of Physical Chemistry A 101 (7) (1997) 1267-1269, doi:10.1021/jp962714v. 